Service social

\title{
Bénéficiaires et intervenants, partenaires dans l'amélioration de la qualité de vie : une recherche-action en milieu de soins prolongés
}

\section{Lise Darveau-Fournier}

Volume 34, numéro 1, 1985

Personnes âgées, milieux de vie et pratiques sociales

URI : https://id.erudit.org/iderudit/706249ar

DOI : https://doi.org/10.7202/706249ar

Aller au sommaire du numéro

Éditeur(s)

École de service social de l'Université Laval

ISSN

1708-1734 (numérique)

Découvrir la revue

Citer cet article

Darveau-Fournier, L. (1985). Bénéficiaires et intervenants, partenaires dans

l'amélioration de la qualité de vie : une recherche-action en milieu de soins

prolongés. Service social, 34(1), 30-45. https://doi.org/10.7202/706249ar 
DARVEAU-Fournier, Lise, professeur à l'École de service social de l'Université Laval.

\section{Bénéficiaires et intervenants, partenaires dans l'amélioration de la qualité de vie : une recherche-action en milieu de soins prolongés}

\section{Lise Darveau-Fournier}

L'amélioration de la qualité de vie constitue un thème dont on discute beaucoup dans les milieux de la santé et des services sociaux, particulièrement depuis la dernière décennie. La vie en institution fait l'objet de questionnement et de recherche de solutions qu'on voudrait novatrices et porteuses de changements, tant au niveau du développement ou du maintien de l'autonomie des bénéficiaires qu'à celui des relations interpersonnelles entre les instances concernées (Conseil des affaires sociales et de la famille, 1978). Ce même Conseil souligne que, parmi les clientèles qui requièrent des soins de santé, celle des soins prolongés est peut-être la plus vulnérable et la plus démunie.

Nous présentons, dans cet article, la nature et les résultats d'un projet de recherche-action mené en milieu de soins prolongés, de 1982 à 1984, par une équipe interdisciplinaire de l'Université Laval, ${ }^{*}$ en collaboration avec les autorités concernées et avec le support financier du Ministère des affaires sociales du Québec.

\section{Nature et contexte du projet}

Des bénéficiaires de soins prolongés, des membres de leur famille et les diverses catégories d'intervenants de l'Hôpital Général de Québec ont travaillé ensemble à la préparation du projet puis à l'implantation d'un programme de formation continue, par les pairs, dans leur milieu même, en vue d'améliorer la qualité de vie dans l'institution. 
L'Hôpital Général constitue l'un des plus anciens milieux hospitaliers de Québec; ses origines remontent à 1693, soit au début de la colonie. Après avoir cumulé diverses vocations, entre autres hôpital, asile, école d'infirmières auxiliaires, il comprend actuellement un centre hospitalier de soins prolongés et un centre d'accueil regroupant respectivement 292 et 142 bénéficiaires. Sur l'ensemble, soit 434 personnes au moment de l'étude, $83.2 \%$ ont 75 ans et plus, et $90.8 \%$ sont des femmes.

Comme dans la majorité des institutions de ce type, on remarque, depuis quelques années, des changements importants dans les caractéristiques de la clientèle, en particulier, l'élévation de l'âge, avec les conséquences que cela entraîne: détérioration de l'état de santé, perte d'autonomie, besoins accrus d'aide, tant au plan physique que psychosocial. En même temps que s'alourdissent les besoins auxquels doivent répondre les intervenants, il faut aussi faire face à des coupures budgétaires qui se traduisent par une diminution de ressources humaines et matérielles.

Tout contribue à rendre encore plus complexe la tâche des intervenants et à augmenter leur degré d'insatisfaction face à leurs conditions de travail; cela entraîne donc des difficultés au plan des relations entre les intervenants eux-mêmes et aussi avec les bénéficiaires.

Le moyen retenu pour faciliter un ressourcement des diverses catégories de personnes qui forment la communauté de l'Hôpital Général a été la formation continue par les pairs en milieu de travail. Cette démarche diffère des modèles traditionnels par les deux aspects suivants : d'abord, ce sont les pairs qui identifient le contenu sur lequel porteront les activités, puis qui discutent ensemble des moyens concrets d'améliorer leur pratique quotidienne; de plus, ces activités se déroulent dans le milieu même, pendant les heures habituelles de travail. Les formules traditionnelles sont ordinairement axées sur la transmission de connaissances par un professeur considéré comme un expert dans le domaine concerné; les cours sont habituellement dispensés en dehors des lieux de travail et regroupent, la plupart du temps, des individus qui œuvrent dans des organismes différents et qui risquent donc de présenter moins de points d'intérêt communs.

Hickey (1974) met en évidence une certaine inefficacité de ce modèle traditionnel et insiste sur l'importance de développer des formules qui correspondent davantage aux besoins des adultes en milieu de travail. Hortala (1979) préconise une formation qui tient compte du contexte et des besoins spécifiques d'un milieu de travail ; elle suggère de réunir "au même moment, plusieurs travailleurs d'une 
même équipe, voire une équipe en son entier quand c'est possible» (p. 38).

Les résultats d'une étude menée par Almquist, Stein et leurs collaboratrices (1981) leur permettent de croire que "la formation continue constitue un moyen efficace d'influencer les connaissances et les attitudes du personnel de soins prolongés et on devrait s'en servir comme première étape vers une amélioration de la qualité des soins aux bénéficiaires âgés" (p. 117).

C'est dans cet esprit, et en s'inspirant d'expériences tentées récemment par d'autres chercheurs de l'Université Laval (Tremblay, 1981 ; Bibeau, Rhéault-Turgeon et al., 1982), que le programme a été conçu et réalisé à l'Hôpital Général. Il a aussi été appliqué par la même équipe de recherche, et à peu près au même moment, mais avec certaines particularités au plan de la méthodologie, au Centre hospitalier Saint-Augustin, autre institution de soins prolongés dans la région de Québec (Trudel, Lemay et al., 1984).

Le modèle expérimenté tient compte des caractéristiques des adultes qu'il veut rejoindre et fait appel à leurs connaissances et à leur expérience pour orienter les activités de développement à privilégier. II les considère comme des experts en leur domaine et les invite à se prononcer eux-mêmes sur les principes théoriques qu'ils jugent importants d'actualiser dans leur pratique quotidienne auprès des bénéficiaires de soins prolongés. On peut alors le qualifier de modèle andragogique.

Une des caractéristiques spécifiques du projet réside dans la définition élargie que l'on a donné au concept de pairs. On les définit habituellement comme ceux qui partagent une même formation, un même travail, une même situation. Pour les fins du projet, nous l'avons défini dans le sens où Fordyce (1981) l'indique, c'est-à-dire en le situant dans un processus d'interdisciplinarité. Nous avons donc considéré comme "pairs" toutes les personnes, cadres, intervenants de formations diverses, bénéficiaires et leur famille, qui œuvrent ensemble à l'amélioration de la qualité de vie à l'Hôpital Général.

\section{Objectifs visés}

"Améliorer la qualité de vie des bénéficiaires et la satisfaction au travail des intervenants par un processus de formation continue», tel est le but explicité dans le programme implanté à l'Hôpital Général. Les principaux objectifs poursuivis visent à "stimuler le développement d'attitudes qui favorisent une approche globale du bénéficiaire" et "à 
valoriser la contribution particulière des intervenants et des bénéficiaires au sein de l'équipe multidisciplinaire." (Darveau-Fournier, Chalifour, Goulet et al., 1985, p. 15).

On peut définir l'approche globale comme étant celle qui préconise une perspective unifiée de la personne, c'est-à-dire qui tient compte, à la fois de ses besoins physiologiques, psychosociaux et spirituels (Burnside, 1978; Bennett, 1978; Conseil des affaires sociales et de la famille, 1978). Elle favorise également une participation active du bénéficiaire dans les décisions qui le concernent et dans une prise en charge de ses besoins, à la mesure de ses capacités. Une telle philosophie d'intervention va à l'encontre du modèle médical qui préconise plutôt une prise en charge totale par l'institution de soins.

L'approche globale ne peut se réaliser sans une collaboration étroite et constante de toutes les parties concernées, y compris le bénéficiaire lui-même. L'équipe interdisciplinaire, que Rothberg (1981, p. 408) définit comme un groupe de personnes qui possèdent chacune une expertise particulière et qui partagent un but et des objectifs communs, s'avère alors le lieu privilégié où peut se développer la concertation indispensable à ce type d'approche (C.A.S.F., 1978).

\section{Méthodologie}

La méthodologie retenue faisait appel à la participation de toutes les catégories de personnes formant la communauté de l'Hôpital Général: cadres, personnel soignant, personnel de soutien, bénéficiaires et leur famille, et ce, à diverses étapes du projet. Le tableau qui suit illustre ces étapes, de même que les modalités spécifiques à chacune et les acteurs qui y sont principalement impliqués (Tableau 1).

\section{a) Consultation sur les critères de qualité d'intervention}

Cette première étape s'adressait à l'ensemble de la population de l'Hôpital Général et avait pour but d'identifier les principes et les modalités d'intervention qu'une majorité de répondants jugeaient importants pour assurer une meilleure qualité de vie dans leur institution.

Elle s'est faite au moyen d'un questionnaire qui contenait cent quarante-trois énoncés, tirés d'une étude de documentation et regroupés autour de thèmes tels que la philosophie des soins prolongés, l'environnement, les activités de la vie quotidienne, les dimensions psychosociales, certaines questions spécifiques sur l'incontinence, la 


\section{TABLEAU 1}

\section{Étapes du programme de formation *}

\begin{tabular}{|c|c|c|}
\hline Étapes & Modalités & Acteurs \\
\hline 1. Consultation & $\begin{array}{l}\text { Rencontres d'infor- } \\
\text { mation sur le projet } \\
\text { Questionnaire de } \\
\text { consultation sur les } \\
\text { critères de qualité } \\
\text { d'intervention ( } 3 \text { tours) } \\
\text { Liste consensuelle des } \\
\text { critères de qualité } \\
\text { d'intervention }\end{array}$ & $\begin{array}{l}\text {-Personnel soignant et } \\
\text { de soutien, bénéfi- } \\
\text { ciaires et leur famille } \\
\text { et équipe de } \\
\text { recherche } \\
\text {-Participants volon- } \\
\text { taires (suite à l'infor- } \\
\text { mation reçue sur le } \\
\text { projet) } \\
\text {-Équipe de recherche } \\
\text { (à partir des réponses } \\
\text { aux trois tours de } \\
\text { consultation) }\end{array}$ \\
\hline $\begin{array}{l}\text { 2. Confrontation } \\
\text { des critères de } \\
\text { qualité d'inter- } \\
\text { vention avec la } \\
\text { pratique }\end{array}$ & $\begin{array}{l}\text { Choix des critères à } \\
\text { discuter en groupe } \\
\text { Rencontres de dis- } \\
\text { cussion de groupe } \\
\text { incluant les séances de } \\
\text { théâtre par images }\end{array}$ & $\begin{array}{l}\text {-Groupe nominal } \\
\text { formé de représen- } \\
\text { tants des participants } \\
\text {-Équipe de recherche, } \\
\text { participants, anima- } \\
\text { trices de groupe, } \\
\text { animateurs du théâtre } \\
\text { par images }\end{array}$ \\
\hline $\begin{array}{l}\text { 3. Évaluation du } \\
\text { programme de } \\
\text { formation }\end{array}$ & $\begin{array}{l}\text { Évaluation des étapes } 1 \\
\text { et } 2 \text { à partir de: } \\
\text { - guide de discus- } \\
\text { sion } \\
\text { - enregistrement } \\
\text { des rencontres de } \\
\text { groupe } \\
\text { - rapports des } \\
\text { animatrices } \\
\text { Questionnaires (pré- } \\
\text { test et post-test) sur: } \\
\text { - les attitudes } \\
\text { - la satisfaction au } \\
\text { travail } \\
\text { - l'environnement }\end{array}$ & $\begin{array}{l}\text {-Participants, anima- } \\
\text { trices et équipe de } \\
\text { recherche }\end{array}$ \\
\hline
\end{tabular}

Source : Darveau-Fournier, Chalifour, Goulet et al., 1985, pp. 17-18. 
confusion et la mort et le fonctionnement en équipe multidisciplinaire. Les répondants étaient invités à indiquer leur accord ou leur désaccord pour chacun des énoncés ou critères proposés ; lorsqu'ils mentionnaient un désaccord, ils pouvaient suggérer les modifications qui le leur rendraient plus acceptable. Ils pouvaient aussi en suggérer de nouveaux. Ces derniers, de même que ceux qui avaient fait l'objet de modifications, étaient soumis à un deuxième puis à un troisième tour de consultation. Un critère était reconnu comme ayant obtenu un consensus lorsqu'il recueillait l'accord d'au moins $90 \%$ des répondants.

Cette façon de procéder s'inspire de la technique Delphi, laquelle fait appel à des individus reconnus "experts" dans leur domaine de compétence pour dégager leurs opinions consensuelles sur des sujets spécifiques et s'en servir comme des éléments de théories (Linstone et Turoff, 1975 ; Ouellet-Dubé, 1983). Dans le cadre du projet de l'Hôpital Général, nous avons considéré comme des "experts" toutes les personnes directement impliquées dans la vie de l'institution, y compris les bénéficiaires et leur famille. Les résultats de la consultation ont permis d'élaborer une liste consensuelle de critères de qualité d'intervention, fournissant ainsi le contenu de la deuxième étape : leur confrontation avec la pratique quotidienne.

b) Confrontation des critères de qualité d'intervention avec la pratique quotidienne

La discussion en groupes interdisciplinaires, c'est-à-dire formés d'intervenants de formations et de fonctions différentes ainsi que de bénéficiaires et de membres de leur famille, a été retenue comme moyen privilégié de s'interroger sur la vie à l'institution, à partir des conclusions de la consultation, et de trouver ensemble des actions à entreprendre pour provoquer des changements dans les attitudes, les comportements, les règles et procédures ou dans l'aménagement des lieux et des conditions de vie.

Neuf critères, parmi les cent soixante-trois ayant fait l'objet d'un consensus lors de l'étape de consultation, ont été sélectionnés par un groupe représentatif de l'ensemble des participants, en s'inspirant de la technique du groupe nominal décrite par Van de Ven et Delbecq (1972). Ils ont fait l'objet des discussions des groupes interdisciplinaires.

\section{c) Évaluation}

L'évaluation proposée avait pour but d'identifier les effets du programme de formation continue sur les participants, en termes de 


\section{TABLEAU 2}

\section{Instruments de cueillette des données}

\begin{tabular}{|c|c|c|}
\hline Objet d'évaluation & Instruments & Moment d'utilisation \\
\hline $\begin{array}{l}\text { Changements dans: } \\
\text { - les attitudes des participants } \\
\text { - le degré de satisfaction au } \\
\text { travail } \\
\text { - la perception de l'environ- } \\
\text { nement }\end{array}$ & $\left.\begin{array}{c}\text { Questionnaire de type objectif comprenant: } \\
\text { - l'échelle d'attitude du personnel soignant } \\
\text { envers le potentiel de réadaptation des } \\
\text { personnes âgées } \\
\text { - l'échelle de satisfaction au travail } \\
\text { - l'échelle de qualité de l'environnement en } \\
\text { milieu de soins prolongés }\end{array}\right\}$ & $\begin{array}{l}\text { Au début de l'étape "Consultation" } \\
\text { et à la fin de l'étape "Confrontation" }\end{array}$ \\
\hline $\begin{array}{l}\text { Perceptions et commentaires sur: } \\
\text { - la formule pédagogique } \\
\text { - le contenu théorique } \\
\text { - la pertinence du projet }\end{array}$ & $\begin{array}{l}\text { Guide de discussion } \\
\text { et } \\
\text { Rapports des animatrices }\end{array}$ & $\begin{array}{l}\text { Dernière rencontre des groupes de } \\
\text { discussion et après chaque rencontre } \\
\text { des groupes de discussion }\end{array}$ \\
\hline
\end{tabular}


changements dans leurs attitudes, leur degré de satisfaction au travail et leur perception de l'environnement immédiat, c'est-à-dire l'hôpital. Elle visait aussi à recueillir leurs perceptions et leurs commentaires sur la formule pédagogique utilisée, le contenu théorique véhiculé, ainsi que sur la pertinence du projet dans son ensemble.

Le tableau 2 identifie les instruments de cueillette de données pour chacun des volets de l'évaluation, de même que le moment de leur utilisation.

Nous avons utilisé l'échelle d'attitude du personnel soignant envers le potentiel de réadaptation des personnes âgées (D'Amour, 1978) et celle sur la satisfaction au travail (Brayfield et Rothe, 1951), qui comprennent chacune dix-huit énoncés sur lesquels les répondants expriment leur degré d'accord à l'aide d'une échelle qualitative et l'échelle sur la qualité de l'environnement en milieux de soins prolongés (Moos, 1977) contenant soixante-dix questions fermées auxquelles on répondait par oui ou non.

\section{TABLEAU 3}

\section{Sujets discutés par thème}

\begin{tabular}{|c|c|}
\hline Thème & Sujet traité \\
\hline Activités de la vie quotidienne & $\begin{array}{l}\text { - autonomie du bénéficiaire } \\
\text { - alimentation du bénéficiaire }\end{array}$ \\
\hline Environnement physique & $\begin{array}{l}\text { - ressemblance entre l'environne- } \\
\text { ment de l'hôpital et de la maison } \\
\text { - adaptation aux besoins physiques } \\
\text { du bénéficiaire }\end{array}$ \\
\hline Dimensions psychosociales & $\begin{array}{l}\text { - façon de s'adresser au bénéfi- } \\
\text { ciaire } \\
\text { - intimité des bénéficiaires }\end{array}$ \\
\hline $\begin{array}{l}\text { Questions spécifiques: } \\
\text { - incontinence }\end{array}$ & $\begin{array}{l}\text { - respect et dignité de l'inconti- } \\
\text { nent }\end{array}$ \\
\hline - confusion & $\begin{array}{l}\text { - place de la personne confuse } \\
\text { par rapport aux autres bénéfi- } \\
\text { ciaires }\end{array}$ \\
\hline - mort & - accompagnement du mourant \\
\hline
\end{tabular}

Source : Darveau-Fournier, Chalifour, Goulet et al., 1985, p. 37. 
Le guide de discussion proposé pour permettre l'expression des perceptions et commentaires des participants sur l'ensemble du projet a été préparé par l'équipe de recherche pour l'usage des animatrices des rencontres de discussion. Quant aux rapports des animatrices, ils devaient contenir, pour chaque rencontre, le résumé des discussions, les suggestions émises et une courte évaluation de la rencontre.

\section{Résultats}

Le projet de formation continue par les pairs a rejoint un nombre impressionnant de membres de la communauté de l'Hôpital Général. Environ trois cent cinquante personnes, incluant des intervenants de formations et de fonctions diverses, travaillant de jour, de soir ou de nuit, des bénéficiaires, des membres de leur famille et des bénévoles, ont assisté aux rencontres d'information tenues à l'Hôpital pendant les heures régulières de travail et ont été invitées à participer au projet.

\section{Consultation sur les critères de qualité d'intervention}

Deux cent soixante-dix-neuf personnes ont rempli le questionnaire sur les critères de qualité d'intervention lors du premier tour de consultation. La compilation des réponses aux trois tours, tel que spécifié dans la méthodologie, a permis d'en identifier cent soixantetrois comme normes idéales de pratique par au moins $90 \%$ des répondants et de les consigner dans une liste consensuelle. Cette liste contient ceux qui ont fait l'objet d'un consensus, ceux qui ont été rejetés, les pourcentages d'accord obtenus de même que les résumés de littérature qui les accompagnent (Darveau-Fournier, Chalifour, Goulet et al., 1985, pp. 107-177).

\section{Confrontation des critères de qualité d'intervention avec la pratique}

La confrontation des critères avec la pratique quotidienne s'est réalisée à travers dix-sept groupes interdisciplinaires de discussion qui se sont réunis chacun à cinq reprises sur une période de trois mois.

Les participants inscrits aux groupes de discussion se répartissaient de la façon suivante : les infirmières et infirmiers auxiliaires étaient les plus nombreux, suivis des préposés aux bénéficiaires, des infirmières et 
infirmiers, des préposés au service alimentaire, des préposés à l'entretien, des bénéficiaires, des administrateurs (coordonnatrices et infirmières-chefs), des techniciens (en diététique, réadaptation et autres), des autres professionnels (ergothérapeutes, physiothérapeutes, diététiciennes, aumôniers) et des parents de bénéficiaires.

Quatre des rencontres ont été animées par des personnesressources dont le rôle était surtout d'en faciliter le progrès et la participation de chaque membre. L'autre s'est déroulée sous forme d'un théâtre-image, tel que développé en Amérique latine par Boal (1983) et adapté au Québec par des membres du "Théâtre sans détour".

Sur les 259 participants, 213 ont assisté à la première rencontre alors que 137 ont persévéré jusqu'à la cinquième. Le tableau 3 présente la liste des sujets discutés. Les participants ont donné, sur chacun d'eux, leur point de vue; ils ont identifié des difficultés qu'ils rencontraient et ont émis des suggestions concrètes de nature à améliorer les conditions de vie des bénéficiaires ou des intervenants. Une synthèse des discussions est consignée dans le Rapport final de la recherche (Darveau-Fournier, Chalifour, Goulet et al., 1985, pp. 41-54).

\section{Évaluation}

Trois cent vingt-cinq personnes ont répondu au questionnaire d'évaluation initiale alors que cent vingt-cinq ont complété le questionnaire d'évaluation finale, lequel visait à identifier les modifications au niveau des attitudes, de la satisfaction au travail et de l'environnement. Cent trente-sept personnes ont assisté à la cinquième rencontre au cours de laquelle on a recueilli, à partir du guide de discussion, les perceptions des participants sur l'ensemble du projet, particulièrement sur la formule pédagogique utilisée et sur les apprentissages réalisés.

\section{a) Perceptions des participants sur l'ensemble du projet}

La consultation sur les critères de qualité d'intervention a permis de réfléchir sur ses diverses facettes en milieu de soins prolongés et sur leur propre façon de travailler, selon plusieurs participants. Par contre, un certain nombre ont fait remarquer qu'ils avaient trouvé difficile de répondre à un questionnaire qui portait sur des thèmes complexes et qui contenait, au premier tour, en plus des résumés de littérature qui les accompagnaient, cent quarante-trois critères. Au sujet de leur 
confrontation avec la pratique, la plupart des participants disent avoir beaucoup apprécié les rencontres interdisciplinaires, lesquelles leur ont fourni une occasion de se parler entre personnes de formations et de fonctions différentes, de comprendre davantage les rôles de chacun, les difficultés que rencontrent d'autres équipes (de jour, de soir ou de nuit) ou d'autres départements et les solutions que chacun y apporte, de se sentir davantage solidaires et complémentaires les uns des autres. Pour plusieurs, elles ont été une occasion de repenser la routine quotidienne et de se questionner à nouveau sur les besoins des bénéficiaires. La présence de ces derniers a d'ailleurs été appréciée par la plupart des participants. La majorité a souligné le climat de chaleur, d'égalité et de liberté d'expression qui régnait et a dit s'être sentie à l'aise dans les groupes.

Même s'ils n'ont pas acquis de nouvelles connaissances techniques comme telles, la plupart considèrent qu'ils ont"commencé à développer un nouvel esprit dans lequel effectuer leur travail ». Ils souhaitent que la démarche de formation amorçée se poursuive et soit même renforcée; certains suggèrent que des rencontres interdisciplinaires deviennent obligatoires pour tous et que des mesures incitatives soient mises en place, entre autres que les infirmières-chefs et les membres de la direction y participent, que la charge de travail tienne compte de telles activités et que des locaux adéquats soient disponibles. La plupart considèrent que le travail d'équipe devrait se développer à divers paliers : au niveau des départements d'abord, dans le but de dépasser le simple objectif d'information et permettre l'expression d'opinions, de désirs, de frustrations; puis au niveau des équipes qui interviennent auprès des mêmes bénéficiaires, mais selon des horaires différents, afin d'assurer une participation active de chacun et une continuité dans les plans des soins. On souhaite également que des rencontres occasionnelles entre membres de départements différents se poursuivent afin de profiter des idées et des expériences des autres pour se stimuler et travailler ensemble à l'amélioration de la qualité de vie du milieu.

\section{b) Changements au niveau des attitudes, de la} satisfaction au travail et de l'environnement

Les données proviennent de cent questionnaires tirés au hasard parmi les trois cent vingt-cinq de l'évaluation initiale et de cent questionnaires parmi les cent vingt-cinq de l'évaluation finale. Chaque sous-test a été traité séparément et les données ont été soumises à une analyse informatisée selon le système S.P.S.S. (Statistical Package in Social Sciences). Le test " $F$ » a été utilisé pour comparer la différence de 
moyennes des résultats obtenus avant et après la formation; la probabilité d'erreur a été fixée à 05 .

Les résultats obtenus pour le premier sous-test, portant sur l'attitude du personnel soignant envers le potentiel de réadaptation des personnes âgées, démontrent un changement statistiquement significatif ( $P$ : 0.014) au niveau des attitudes des participants entre le début et la fin du programme de formation. Le graphique qui suit démontre visuellement ces résultats.

\section{GRAPHIQUE}

\section{Comparaison des résultats au test d'attitude avant et après le programme de formation}

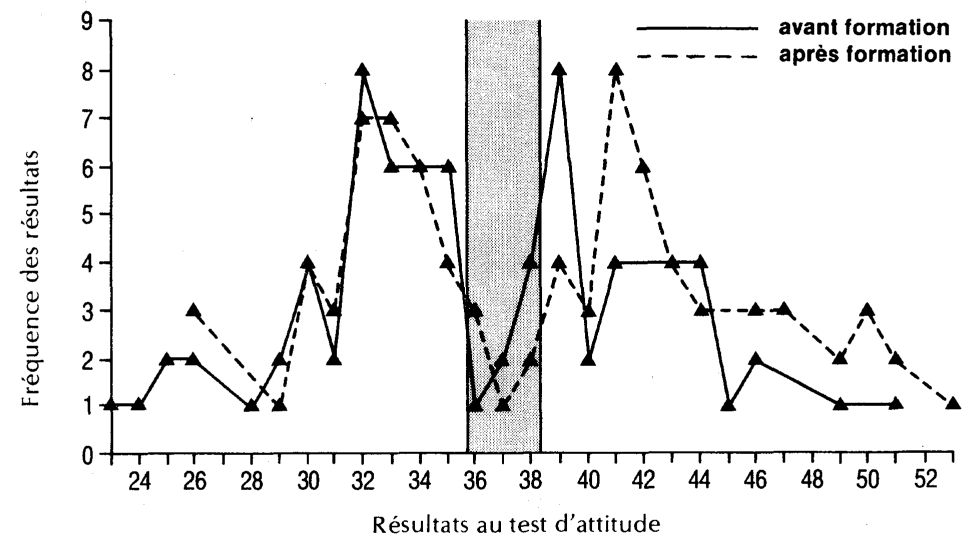

Source: Darveau-Fournier, Chalifour, Goulet et al., 1985, p. 66.

L'analyse des deux autres sous-tests, qui portaient sur la satisfaction au travail et sur l'environnement, ne permet pas de faire ressortir des différences significatives ( $P$ : 0,7825 pour le $2^{\mathrm{e}}$ sous-test et $\mathrm{P}: 0,9157$ pour le $3^{\mathrm{e}}$ ) dans les réponses des participants avant et après la formation. Ces résultats peuvent s'expliquer, entre autres, par la période relativement courte qui s'est écoulée entre les deux mesures, soit moins d'un an ; le programme de formation aurait aussi contribué à rendre les participants plus critiques par rapport aux besoins et aux conditions du milieu. Il est possible également que les participants n'aient pas encore réussi à modifier des aspects concrets de la pratique 
et que les administrateurs ne soient pas encore intervenus pour mettre en place des actions précises dans le sens des suggestions émises par les groupes.

\section{Commentaires}

Une des caractéristiques principales du programme de formation continue expérimenté à l'Hôpital Général de Québec réside dans le nombre et la diversité des personnes qu'il a regroupées de même que dans la place qu'il leur a permis de prendre dans une action concertée en vue d'améliorer la qualité de vie, tant des bénéficiaires que des intervenants. L'étape "consultation" a fourni à au moins 194 membres du personnel et 34 bénéficiaires dûment identifiés, parmi les 279 répondants, l'occasion de s'interroger sur la vie en contexte de soins prolongés et de donner leur opinion sur les critères de qualité d'intervention qu'ils jugent importants. Une telle démarche peut aider les participants à réaliser qu'ils sont des composantes importantes, ont des connaissances et une expérience qui en font des experts dans leur domaine et, qu'en s'engageant activement dans une démarche collective, ils peuvent contribuer à améliorer leur propre situation et celle du milieu. La liste consensuelle, établie à partir des résultats de la consultation, constitue une banque de données qui correspond aux aspirations des diverses catégories de personnes qui forment la communauté de l'Hôpital Général ; elle peut fournir du matériel de base pour la poursuite d'activités de formation continue durant plusieurs années à venir.

L'étape "confrontation des critères avec la pratique quotidienne" a permis à au moins 137 personnes, qui provenaient de presque toutes les catégories d'intervenants et de bénéficiaires, d'amorcer une conception renouvelée de l'intervention, soit l'expérimentation d'une collaboration interdisciplinaire en vue d'une approche globale des besoins des diverses parties concernées. Une telle approche est de nature à redonner au bénéficiaire la place qui lui revient dans sa propre prise en charge, à la mesure de ses capacités, en même temps que d'impliquer activement dans l'orientation et le fonctionnement de l'institution toutes les catégories de personnel qui y œuvrent.

Les neuf sous-thèmes discutés au cours des rencontres de groupe (voir tableau 3) ont donné lieu à des suggestions appropriées et réalisables qui concernent, entre autres, le domaine des attitudes (en rapport avec le respect des habitudes de vie, des capacités et de la dignité de la clientèle, de la confidentialité, etc.), celui des règles et procédures de fonctionnement (lors de l'accueil, pour des modalités 
de travail en équipe, etc.), celui de l'utilisation des ressources humaines (les familles, les bénévoles, le personnel requis à certaines périodes de la journée, etc.), celui de l'aménagement physique et matériel (pour les bénéficiaires moins autonomes, pour ceux en chaises roulantes, pour l'aménagement de lieux de rencontres adaptés aux besoins, etc.).

La formation des intervenants a fait l'objet de suggestions spécifiques qui concernent particulièrement la préparation des nouveaux membres du personnel, l'approche aux mourants, la formation continue par les pairs.

Certaines difficultés ont été rencontrées au cours du déroulement du projet ; plusieurs étaient d'ordre organisationnel et ont contribué à la diminution du nombre de participants aux discussions (de 213 à la première rencontre, le nombre est passé à 137 à la cinquième). Parmi les principales raisons invoquées, mentionnons l'absence de libération complète des intervenants aux moments opportuns: la somme de travail à accomplir demeurait la même et certains devaient quitter avant la fin des réunions pour effectuer des tâches précises sur le département, avant le changement de l'équipe de travail. De plus, pour les infirmières-chefs et les coordonnatrices, ces activités ont coïncidé avec une évaluation de chacun des bénéficiaires dans le cadre d'une opération du Ministère des affaires sociales. Les horaires de travail qui varient d'un mois à l'autre ont aussi occasionné des absences étant donné les jours de congé des intervenants, qui coïncidaient parfois avec les jours de rencontres.

Malgré tout, on peut considérer que le programme de formation continue par les pairs a donné des résultats positifs à l'Hôpital Général. Il a contribué à réunir les diverses composantes du milieu, intervenants et bénéficiaires, dans une recherche concertée pour améliorer la qualité de vie à l'hôpital; on peut aussi raisonnablement croire qu'il a permis une modification des attitudes du personnel envers le potentiel de réadaptation des personnes âgées, tel que le démontrent les résultats de l'évaluation.

Le programme réalisé ne constitue toutefois qu'une amorce de formation. Quelques critères seulement ont été étudiés ; de plus, tous les intervenants n'ont pas eu l'occasion de confronter leur pratique avec les critères idéaux de qualité d'intervention. L'enthousiasme des participants et leur désir de continuer la démarche entreprise constitue un atout important pour la poursuite de la formation; le matériel de discussion est disponible et c'est maintenant à la Direction de l'hôpital d'assurer une continuité, entre autres en s'impliquant dans l'orientation de la formation continue à partir des besoins et des suggestions déjà 
émises et en mettant en place des conditions qui facilitent la participation de chacun.

\section{Note}

* L'équipe de recherche était composée de: Olive Goulet, directrice du projet, et Jacques Chalifour de l'École des sciences infirmières, Lise DarveauFournier de l'École de service social, Lise Lemay du Département de médecine sociale et préventive et Louis Trudel de l'École de réadaptation, assistés de Normand Leavy, chargé de recherche.

\section{Références bibliographiques}

Almquist, E., S. Stein, A. Weiner et M.W. LinN, "Evaluation of continuing education for long-term care personnel: impact upon attitudes and knowledge ", Journal of the American Geriatrics Society, vol. 29, $n^{\circ} 3,1981$ : 117-122.

BENNET, B.K. "Humanizing long-term care", Dimensions in Health Services, vol. 55, $\mathrm{n}^{\circ}$ 5, 1978: 15-18.

Bibeau, G., L. Rheault-Turgeon, G. Deschênes, M. Laverkene, N. Leavy et C. Poulin, Perspectives pour une amélioration de la qualité des services au Centre François-Charon, "Rapport final du projet expérimental de formation continue, 1980-1981", Québec, Université Laval, 1982.

BOAL, A., Théâtre de l'opprimé, Paris, Maspero, 1983.

BRAYfield, A.H. et H.F. Rothe, "An index of job satisfaction ", Journal of Applied Psychology, vol. 35, $n^{\circ}$ 5, $1951:$ 307-311.

BURNSIDE, I.M. (éd.), Working with the Elderly: Group Processes and Techniques, North Scituate (Mass.), Duxbury Press, 1978.

Conseil des affaires sociales et de la famille, Les objectifs et les moyens susceptibles d'améliorer la situation des malades chroniques, Québec, Éditeur officiel du Québec, 1978. ("Études et avis".)

Darveau-Fournier, L., J. Challfour, O. Goulet, L. Trudel, L. Lemay et N. Leavy, Formation continue par les pairs, Hôpital Général de Québec (1982-1984). Rapport final, Québec, Université Laval, 1985.

D'Amour, D., Attitudes du soignant envers le potentiel de réadaptation des malades âgés : étude méthodologique, mémoire présenté à la Faculté des études supérieures de l'Université de Montréal en vue de l'obtention d'une maitrise, 1978.

FordycE, W.E., "On interdisciplinary peers", Archives of Physical Medicine and Rehabilitation, vol. 62, $\mathrm{n}^{\circ} 2,1981: 51-53$.

HICKEY, T., "In-service training in gerontology : toward the design of an effective educational process", The Gerontologist, vol. 14, $\mathrm{n}^{\circ} 1,1974:$ 54-64. 
Hortala, F., "Formation et soutien au personnel engagé dans l'action gériatrique», Gérontologie, $\mathrm{n}^{\circ} 29,1979$ : 26-39.

Linstone, H.A. et M. Turoff (éds.), The Delphi Method: Techniques and Applications, Don Mills, Addison-Wesley, 1975.

Moos, R.H., Sheltered Care Project, Social Ecology Laboratory, Veterans Administration Hospital and Standford University Medical Center, Palo Alto (Calif.), 1977.

Ouellet-Dubé, F., "La technique Delphi : sa nature, son utilité, son utilisation"; Mesure et évaluation en éducation, vol. 6, $\mathrm{n}^{\circ} 1,1983: 3-15$.

ROTHBERG, J.S., "The rehabilitation team : future direction", Archives of Physical Medicine and Rehabilitation, vol. 62, $n^{\circ} 8,1981: 407-410$.

Tremblay, J., "Creating an appropriate climate for drug use review", American Journal of Hospital Pharmacy, vol. 38, $\mathrm{n}^{\circ}$ 3, 1981 : 212-215.

Trudel, L., L. Lemay, O. Goulet, l. Darveau-Fournier, J. Challfour, et N. Leavy, Programme de formation continue par les pairs au Centre hospitalier Saint-Augustin (1982-1984). Rapport final, Québec, Université Laval, 1984.

VAN DE VEN, A. et A. DELBEQ, "The nominal group as a research instrument for exploratory health studies ", American Journal of Public Health, vol. 62, no 3, 1972 : 337-342. 